\title{
The Generalized Search for a Randomly Moving Target
}

\author{
Abdelmoneim Anwar Mohamed Teamah \\ Mathematics Department, Faculty of Science, Tanta University, Tanta, Egypt \\ Email: teamah4@hotmail.com
}

Received 12 September 2013; revised 12 October 2013; accepted 19 October 2013

Copyright (C) 2014 by author and Scientific Research Publishing Inc.

This work is licensed under the Creative Commons Attribution International License (CC BY). http://creativecommons.org/licenses/by/4.0/

(c) (i) Open Access

\begin{abstract}
A target is assumed to move randomly on one of two disjoint lines $L_{1}$ and $L_{2}$ according to a stochastic process $\left\{S(t), t \in \mathfrak{R}^{+}\right\}$. We have two searchers start looking for the lost target from some points on the two lines separately. Each of the searchers moves continuously along his line in both directions of his starting point. When the target is valuable as a person lost on one of disjoint roads, or is serious as a car filled with explosives which moves randomly in one of disjoint roads, in these cases the search effort must be unrestricted and then we can use more than one searcher. In this paper we show the existence of a search plan such that the expected value of the first meeting time between the target and one of the two searchers is minimum.
\end{abstract}

\section{Keywords}

Stochastic Process; Expected Value; Linear Search; Optimal Search Plan

\section{Introduction}

The search for lost targets that are either stationary or randomly moving has recently applications, such as: searching for lost persons on roads, the search for a petroleum or gas underground, and so on (see, Abd-Elmoneim [1], Ohsumi [2], El-Rayes and Abd-Elmoneim [3], and Washburn [4]).

When the target to be found is stationary or moves randomly on the real line, this problem is of interest because it may arise in many real world situations (see El-Rayes et al. [5] and Balkhi [6]). Search problems with stationary target on line are well studied (see El-Rayes and Abd-Elmoneim [7], El-Rayes et al. [8], Balkhi [6] [9], Rousseeuw [10], Abd-Elmoneim and Abu-Gabl [11], and Stone [12]). In the case of randomly moving target on the line and the searcher starts search from the origin, a deal of work has been done for deriving conditions for optimal search path which minimizes the effort of finding the target (see El-Rayes et al. [5], Fristedt 
and Heath [13]). If the lost target is a valuable target as a person lost on one of disjoint roads, or is serious as a car filled with explosives which moves randomly in one of disjoint roads, then the effort of the search (the cost of search) must be unrestricted, in these cases using more than one searcher (see Abd El-Moneim et al. [14]). The search problem for a randomly moving target on one of two disjoint lines will be considered, in previous studies (see Abd El-Moneim and Abu-Gabl [15]), using a searcher for each line where each searcher starts looking for the lost target from the origin of his line, but Abd El-Moneim et al. [14] used searchers starting searching for the target from the origin that is the intersection point of these lines. Each of the searchers moves continuously along his line in both directions of the starting point, in both cases the target motion is a Brownian motion. In this article, a target is assumed to move randomly on one of two disjoint lines $L_{1}$ and $L_{2}$ according to a stochastic process $\left\{S(t), t \in \mathfrak{R}^{+}\right\}$, where $\mathfrak{R}^{+}$is the set of real numbers. This stochastic process satisfies the following conditions:

(i) Let $c \geq \mu$, where $\mu$ is the drift of the process and $c$ is a constant. Then for any $t>0$ and for some $\varepsilon>0, \quad P\{S(t) \succ c t\} \leq \frac{1}{2} \varepsilon^{t}$,

(ii) Let $\mu \neq 0, z_{1} \geq z_{2}$ and $t \geq \max \left(z_{1} / \mu, z_{2} / \mu\right)$, then $P\left\{z_{2} \leq S(t) \leq z_{1}\right\}$ is non-increasing with $t$,

(iii) Let $T$ be a stopping time for $S(t)$, then

$$
E|S(T)| \leq \sigma \sqrt{E(T)}+|\mu| E(T),
$$

where $E$ stands for the expectation value and $\sigma^{2}$ is the variance and

(iv) Let

$$
S(n)=\sum_{i=1}^{n} Z_{i}, n \geq 1,
$$

where $\left\{Z_{i}\right\}$ is a sequence of independent identically distributed random variables (i. i. d. r. v.) and

$$
q(j, j+1)=\sum_{n=0}^{\infty} P[-(j+1)<S(n)<-j],
$$

then $q(j, j+1)$ satisfies the renewal theorem.

Two searcher start looking for the target from some point $\phi_{0}$ for the first line $L_{1}$ and $\bar{\phi}_{0}$ for the second line $L_{2}$. We assume that the speeds of the searchers are $v_{1}$ and $v_{2}$. Assume that $T$ is the set of real numbers and $T^{+}$is the non-negative part of $T$. Foe any $t \in T^{+}$, let $S(t)$ be a random variable with $S(0)=0$, and assume that $Z_{o}$ is the initial position of the target to be a random variable and independent of $S(t), t>0$. A search plan $(\Phi, \bar{\Phi})$ with speed $v_{1}, v_{2}$ is defined such that $\phi: T^{+} \rightarrow T$ and $\bar{\phi}: T^{+} \rightarrow T$, respectively, such that

$$
\begin{gathered}
\left|\phi\left(t_{1}\right)-\phi\left(t_{2}\right)\right| \leq v_{1}\left|t_{1}-t_{2}\right| \\
\left|\bar{\phi}\left(t_{1}\right)-\bar{\phi}\left(t_{2}\right)\right| \leq v_{2}\left|t_{1}-t_{2}\right|, \quad \forall t_{1}, t_{2} \in T^{+}, \phi \in \Phi, \bar{\phi} \in \bar{\Phi}
\end{gathered}
$$

the first meeting time is a random variable valued in $I^{+}$which is defined

$$
\tau_{\hat{\phi}}=\inf \left\{t: \phi(t)=X_{o}+S(t) \text { or } \bar{\phi}(t)=Y_{o}+S(t)\right\}
$$

where $Z_{o}=X_{0}$ if the target moves on the first line and $Z_{o}=Y_{0}$ if the target moves on the second line. Let the search plan of two searchers be represented by $\hat{\Phi}=\{\phi, \bar{\phi}\}$, where $\hat{\Phi}$ is the set of all search plans. The problem is to find search plan $\hat{\phi}=\{(\phi, \bar{\phi}), \phi \in \Phi, \bar{\phi} \in \bar{\Phi}\}$, such that $E_{\tau \hat{\phi}}<\infty$ in this case we call $\hat{\Phi}$ is a finite search plan and if $E \tau_{\hat{\phi}^{*}}<E \tau_{\hat{\phi}} \forall \hat{\phi} \in \hat{\Phi}$, where $E$ terms to expectation value, then we call $\hat{\phi}^{*}$ is optimal.

\section{The Search Plans}

Let $\zeta_{1}, \zeta_{2}$ be positive integers and $v_{1}$ and $v_{2}$ are rational numbers such that:

1) $v_{1}>|\mu|$ and $v_{2}>|\mu|$,

2) $\zeta_{1}, \zeta_{2}>1$, such that $C_{1}=v_{1} \frac{\zeta_{1}-1}{\zeta_{1}+1}, C_{2}=v_{2} \frac{\zeta_{2}-1}{\zeta_{2}+1}$,

Now we shall define sequences $\left\{G_{i}\right\}_{i \geq 0},\left\{r_{i}\right\}_{i \geq 0},\left\{H_{i}\right\}_{i \geq 0}$ for the searcher on the first line $L_{1}$, 
$\left\{\bar{G}_{i}\right\}_{i \geq 0},\left\{\bar{r}_{i}\right\}_{i \geq 0},\left\{\bar{H}_{i}\right\}_{i \geq 0}$ for the searcher on the second line $L_{2}$ and search plans with speed 1 as follows.

$$
\begin{aligned}
& G_{i}=\zeta_{1}^{i}-1, r_{i}=(-1)^{i+1} C_{1}\left[G_{i}+1+(-1)^{i+1}\right], H_{i}=r_{i}+\phi_{0}, i \geq 0 \\
& \bar{G}_{i}=\zeta_{2}^{i}-1, \bar{r}_{\mathrm{i}}=(-1)^{i+1} C_{2}\left[\bar{G}_{i}+1+(-1)^{i+1}\right], \bar{H}_{i}=\bar{r}_{i}+\bar{\phi}_{0}, i \geq 0
\end{aligned}
$$

Let $O$ be a finite set of numbers, such that

$$
O=\{1,3,5, \cdots, m\} \text { for } j \in O, i \in I^{+}
$$

we have

$$
\cdots<H_{2}<\phi_{0}<H_{1}<H_{3}<\cdots<H_{j-2}<H_{j}<0<H_{j+2}<\cdots
$$

for the first searcher, and

$$
\cdots<\bar{H}_{2}<\bar{\phi}_{0}<\bar{H}_{1}<\bar{H}_{3}<\cdots<\bar{H}_{j-2}<\bar{H}_{j}<0<\bar{H}_{j+2}<\cdots
$$

for the second searcher

for any $t \in R^{+}$, if $G_{2 i-1} \leq t<G_{2 i}$ then $\phi(t)=\phi_{0}-H_{2 i-1}-\left[t-G_{2 i-1}\right]$

and if $\bar{G}_{2 i-1} \leq t<\bar{G}_{2 i}, \quad 1 \leq i \leq \frac{j+1}{2}$, then $\bar{\phi}(t)=\bar{\phi}_{0}-\bar{H}_{2 i-1}-\left[t-\bar{G}_{2 i-1}\right]$,

if $G_{i} \leq t<G_{i+1}$, then $\phi(t)=H_{i}+(-1)^{i}\left[t-G_{i}\right]$

and if $\bar{G}_{i} \leq t<\bar{G}_{i+1}, i \geq j+1$, then $\bar{\phi}(t)=\bar{H}_{i}+(-1)^{i}\left[t-\bar{G}_{i}\right]$, and

if $G_{2 i} \leq t<G_{2 i+1}$, then $\phi(t)=H_{2 i}-\phi_{o}+\left[t-G_{2 i}\right]$

and $\bar{G}_{2 i} \leq t<\bar{G}_{2 i+1}, \quad 1 \leq i \leq \frac{j-1}{2}$, then $\bar{\phi}(t)=\bar{H}_{2 i}-\bar{\phi}_{o}+\left[t-\bar{G}_{2 i}\right]$

We use the following notations where $k_{1}(t), \bar{k}_{1}(t), k_{2}(t)$ and $\bar{k}_{2}(t)$ are positive functions, $\varphi_{1}=s(t)-k_{1}(t)$, $\tilde{\varphi}_{1}=s(t)+k_{2}(t)$ on the first line and $\varphi_{2}=s(t)-\bar{k}_{1}(t), \quad \tilde{\varphi}_{2}=s(t)+k_{1}(t)$ on the second line.

Lemma 2.1. if $0<a, b<1$ then $a b<a+b$

Theorem 2.1. If $(\phi, \bar{\phi}) \in \hat{\Phi}$ is a search plan, and let $\gamma_{1}, \gamma_{2}$ are measurable induced by the initial position of the target on the first and second line respectively, then $E\left(\tau_{\bar{\phi}}\right)$ is finite if:

$$
\begin{aligned}
& \int_{-\infty}^{\phi_{0}}\left[\sum_{i=1}^{j / 2} \zeta_{1}^{2 i} P\left(\varphi_{1}\left(G_{2 i}\right)<-x\right)+\sum_{i=\frac{j}{2}+1}^{\infty} \zeta_{1}^{2 i} P\left(\tilde{\varphi}_{1}\left(G_{2 i}\right) \leq-x\right) \gamma_{1}\right](\mathrm{d} x), \\
& \int_{\phi_{0}}^{\infty} \sum_{i=1}^{\infty} \zeta_{1}^{2 i+1} P\left(\varphi_{1}\left(G_{2 i+1}\right)>-x\right) \gamma_{1}(\mathrm{~d} x) \\
& \int_{-\infty}^{\bar{\phi}_{0}} \sum_{i=1}^{j / 2} \zeta_{2}^{2 i} P\left(\varphi_{2}\left(\bar{G}_{2 i}\right)<-y\right)+\sum_{i=\frac{j}{2}+1}^{\infty} \zeta_{2}^{2 i} P\left(\tilde{\varphi}_{2}\left(\bar{G}_{2 i}\right) \leq-y\right) \gamma_{2}(\mathrm{~d} y), \\
& \int_{\bar{\phi}_{0}}^{\infty} \sum_{i=1}^{\infty} \zeta_{2}^{2 i+1} P\left(\varphi_{2}\left(\bar{G}_{2 i+1}\right)>-y\right) \gamma_{2}(\mathrm{~d} y) . \\
& \int_{\phi_{0}}^{\infty}\left[\sum_{i=(j+1) / 2}^{\infty} \zeta_{1}^{2 i+1} P\left(\varphi_{2}\left(G_{2 i+1}\right)>-x\right)+\sum_{i=1}^{(j-1) / 2} \zeta_{1}^{2 i} P\left(\tilde{\varphi}_{1}\left(G_{2 i+1}\right) \leq-x\right)\right] \gamma_{1}(\mathrm{~d} x) \\
& \int_{-\infty}^{\phi_{0}} \sum_{i=1}^{\infty} \zeta_{1}^{2 i} P\left(\tilde{\varphi}_{1}\left(G_{2 i}\right)<-x\right) \gamma_{1}(\mathrm{~d} x) \\
& \int_{\bar{\phi}_{0}}^{\infty}\left[\sum_{i=(j+1) / 2}^{\infty} \zeta_{2}^{2 i+1} P\left(\varphi_{2}\left(\bar{G}_{2 i+1}\right)>-y\right)+\sum_{i=1}^{(j-1) / 2} \zeta_{2}^{2 i} P\left(\tilde{\varphi}_{2}\left(\bar{G}_{2 i+1}\right) \leq-y\right)\right] \gamma_{2}(\mathrm{~d} y) \\
& \int_{-\infty}^{\bar{\phi}_{0}} \sum_{i=1}^{\infty} \zeta_{2}^{2 i} P\left(\tilde{\varphi}_{2}\left(\bar{G}_{2 i}\right)<-y\right) \gamma_{2}(\mathrm{~d} y) \text {. }
\end{aligned}
$$




$$
\left.\begin{array}{l}
\int_{-\infty}^{\phi_{0}}\left[\sum_{i=1}^{j / 2} \zeta_{1}^{2 i} P\left(\varphi_{1}\left(G_{2 i}\right)<-x\right)+\sum_{i=\frac{j}{2}+1}^{\infty} \zeta_{1}^{2 i} P\left(\tilde{\varphi}_{1}\left(G_{2 i}\right)<-x\right)\right] \gamma_{1}(\mathrm{~d} x), \\
\int_{\phi_{0}}^{\infty} \sum_{i=1}^{\infty} \zeta_{1}^{2 i+1} P\left(\varphi_{1}\left(G_{2 i+1}\right)>-x\right) \gamma_{1}(\mathrm{~d} x), \\
\int_{\bar{\phi}_{0}}^{\infty}\left[\sum_{i=(j+1) / 2}^{\infty} \zeta_{2}^{2 i+1} P\left(\varphi_{2}\left(\bar{G}_{2 i+1}\right)>-y\right) \sum_{i=1}^{(j-1) / 2} \zeta_{2}^{2 i+1} P\left(\tilde{\varphi}_{2}\left(\bar{G}_{2 i+1}\right)>-y\right)\right] \gamma_{2}(\mathrm{~d} y), \\
\int_{-\infty}^{\bar{\phi}_{0}} \sum_{i=0}^{\infty} \zeta_{2}^{2 i} P\left(\tilde{\varphi}_{2}\left(\bar{G}_{2 i}\right)<-y\right) \gamma_{2}(\mathrm{~d} y)
\end{array}\right\} \quad \text { If } \phi_{0}>0, \bar{\phi}_{0}<0,
$$

And

$$
\left.\begin{array}{l}
\int_{\phi_{0}}^{\infty}\left[\sum_{i=(j+1) / 2}^{\infty} \zeta_{1}^{2 i+1} P\left(\varphi_{1}\left(G_{2 i+1}\right)>-x\right)+\sum_{i=1}^{(j-1) / 2} \zeta_{1}^{2 i+1} P\left(\tilde{\varphi}_{1}\left(G_{2 i+1}\right)<-x\right)\right] \gamma_{1}(\mathrm{~d} x), \\
\int_{-\infty}^{\phi_{0}} \sum_{i=1}^{\infty} \zeta_{1}^{2 i} P\left(\tilde{\varphi}_{1}\left(G_{2 i}\right)<-x\right) \gamma_{1}(\mathrm{~d} x) \\
\int_{-\infty}^{\bar{\phi}_{0}}\left[\sum_{i=1}^{j / 2} \zeta_{2}^{2 i} P\left(\varphi_{2}\left(\bar{G}_{2 i}\right)<-y\right)+\sum_{i=\frac{j}{2}+1}^{\infty} \zeta_{2}^{2 i} P\left(\tilde{\varphi}_{2}\left(\bar{G}_{2 i}\right)<-y\right)\right] \gamma_{2}(\mathrm{~d} y), \\
\int_{\bar{\phi}_{0}}^{\infty} \sum_{i=1}^{\infty} \zeta_{2}^{2 i+1} P\left(\varphi_{2}\left(\bar{G}_{2 i+1}\right)>-y\right) \gamma_{2}(\mathrm{~d} y),
\end{array}\right\} \text { If } \phi_{0}<0, \bar{\phi}_{0}>0
$$

are finite

Proof: The continuity of $S(t), \phi(t)$ and $\bar{\phi}(t)$ imply that if $X_{0}>\phi_{0}$ then $X_{0}+S(t)$ is greater than $\phi(t)$ on the first line until the first meeting, also if $X_{0}<\phi_{0}$ then $X_{0}+S(t)$ is smaller than $\phi(t)$ on the first line until the first meeting the same for the second line by replacing $X_{0}$ by $Y_{0}$ and $\phi_{0}$ by $\bar{\phi}_{0}$. Hence for any $i \geq 0$.

$$
\begin{gathered}
P\left(\tau_{\hat{\phi}}>t\right)=P\left(\tau_{\phi}>t \text { and } \tau_{\bar{\phi}}>t\right)=P\left(\tau_{\phi}>t\right) \cdot P\left(\tau_{\bar{\phi}}>t\right) \\
P\left(\tau_{\phi}>G_{2 i+1}\right) \leq \int_{-\infty}^{\phi_{0}} P\left(X_{0}+s\left(G_{2 i}\right)<H_{2 i} / X_{0}=x\right) \gamma_{1}(\mathrm{~d} x)+\int_{\phi_{0}}^{-\infty} P\left(X_{0}+s\left(G_{2 i+1}\right)>H_{2 i+1} / X_{0}=x\right) \gamma_{1}(\mathrm{~d} x)
\end{gathered}
$$

we get

$$
P\left(\tau_{\phi}>G_{2 i+1}\right) \leq \int_{-\infty}^{\phi_{0}} P\left(\tilde{\varphi}_{1}\left(G_{2 i}\right)<-x\right) \gamma_{1}(\mathrm{~d} x)+\int_{\phi_{0}}^{-\infty} P\left(\varphi_{1}\left(G_{2 i+1}\right)>-x\right) \gamma_{1}(\mathrm{~d} x)
$$

Also

$$
\begin{gathered}
P\left(\tau_{\bar{\phi}}>G_{2 i+1}\right) \leq \int_{-\infty}^{\bar{\phi}_{0}} P\left(\tilde{\varphi}_{2}\left(\bar{G}_{2 i}\right)<-y\right) \gamma_{2}(\mathrm{~d} y)+\int_{\bar{\phi}_{0}}^{-\infty} P\left(\varphi_{2}\left(\bar{G}_{2 i+1}\right)>-y\right) \gamma_{2}(\mathrm{~d} y) \\
P\left(\tau_{\phi}>G_{2 i}\right) \leq \int_{-\infty}^{\phi_{0}} P\left(\tilde{\phi}_{1}\left(G_{2 i}\right)<-x\right) \gamma_{1}(\mathrm{~d} x)+\int_{\phi_{0}}^{\infty} P\left(\phi_{1}\left(G_{2 i-1}\right)>-x\right) \gamma_{2}(\mathrm{~d} x)
\end{gathered}
$$

and

$$
P\left(\tau_{\bar{\phi}}>\bar{G}_{2 i}\right) \leq \int_{-\infty}^{\overline{\phi_{0}}} P\left(\tilde{\varphi}_{2}\left(\bar{G}_{2 i}\right)<-y\right) \gamma_{2}(\mathrm{~d} y)+\int_{\bar{\phi}_{0}}^{\infty} P\left(\varphi_{2}\left(\bar{G}_{2 i-1}\right)>-y\right) \gamma_{1}(\mathrm{~d} y)
$$

Hence

$$
E\left(\tau_{\hat{\phi}}\right) \leq \int_{0}^{\infty} P\left(\tau_{\hat{\phi}}>t\right) \mathrm{d} t
$$


from Lemma 2.1 then we get:

$$
\begin{aligned}
E\left(\tau_{\hat{\phi}}\right) \leq & \int_{0}^{\infty}\left[P\left(\tau_{\phi}>t\right)+P\left(\tau_{\bar{\phi}}>t\right)\right] \mathrm{d} t \leq \sum_{i=0}^{\infty}\left[\int_{G_{i}}^{G_{i+1}} P\left(\tau_{\phi}>G_{i}\right)+\int_{\bar{G}_{i}}^{\bar{G}_{i+1}} P\left(\tau_{\bar{\phi}}>\bar{G}_{i}\right)\right] \mathrm{d} t \\
\leq & \sum_{i=0}^{\infty}\left[\int_{G_{i}}^{G_{i+1}} P\left(\tau_{\phi}>t\right)+\int_{\bar{G}_{i}} P\left(\tau_{\bar{\phi}}>t\right)\right] \mathrm{d} t \leq \sum_{i=0}^{\infty}\left[\left(G_{i+1}-G_{i}\right) P\left(\tau_{\phi}>G_{i}\right)+\left(\bar{G}_{i+1}-\bar{G}_{i}\right) P\left(\tau_{\bar{\phi}}>\bar{G}_{i}\right)\right] \\
= & \lambda_{1} \sum_{i=0}^{\infty}\left(\zeta_{1}^{i+1}-\zeta_{1}^{i}\right) P\left(\tau_{\phi}>G_{i}\right)+\lambda_{2} \sum_{i=0}^{\infty}\left(\zeta_{2}^{i+1}-\zeta_{2}^{i}\right) P\left(\tau_{\bar{\phi}}>\bar{G}_{i}\right) \\
= & \lambda_{1}\left(\zeta_{1}-1\right) \sum_{i=0}^{\infty} \zeta_{1}^{i} P\left(\tau_{\phi}>G_{i}\right)+\lambda_{2}\left(\zeta_{2}-1\right) \sum_{i=0}^{\infty} \zeta_{2}^{i} P\left(\tau_{\bar{\phi}}>\bar{G}_{i}\right) \\
= & \lambda_{1}\left(\zeta_{1}-1\right)\left[P\left(\tau_{\phi}>0\right)+\zeta_{1} P\left(\tau_{\phi}>G_{1}\right)+\zeta_{1}^{2} P\left(\tau_{\phi}>G_{2}\right)+\zeta_{1}^{3} P\left(\tau_{\phi}>G_{3}\right)+\cdots\right] \\
& +\lambda_{2}\left(\zeta_{2}-1\right)\left[P\left(\tau_{\bar{\phi}}>0\right)+\zeta_{2} P\left(\tau_{\bar{\phi}}>\bar{G}_{1}\right)+\zeta_{2}^{2} P\left(\tau_{\phi}>\bar{G}_{2}\right)+\zeta_{2}^{3} P\left(\tau_{\phi}>\bar{G}_{3}\right)+\cdots\right]
\end{aligned}
$$

If $\phi_{0}, \bar{\phi}_{0}<0$, then

$$
\begin{aligned}
& E\left(\tau_{\hat{\phi}}\right) \leq \lambda_{1}\left(\zeta_{1}-1\right)\left[P\left(\tau_{\phi}>0\right)+\zeta_{1} P\left(\tau_{\phi}>G_{1}\right)+\zeta_{1}^{2}\left\{\int_{\phi_{0}}^{\infty} P\left(\tilde{\phi}_{1}\left(G_{1}\right)>-x\right) \gamma_{1}(\mathrm{~d} x)\right.\right. \\
& \left.+\int_{-\infty}^{\phi_{0}} P\left(\tilde{\varphi}_{1}\left(G_{2}\right)<-x\right) \gamma_{1}(\mathrm{~d} x)\right\}+\zeta_{1}^{3}\left\{\int_{\phi_{0}}^{\infty} P\left(\tilde{\varphi}_{1}\left(G_{3}\right)>-x\right) \gamma_{1}(\mathrm{~d} x)+\int_{-\infty}^{\phi_{0}} P\left(\tilde{\phi}_{1}\left(G_{2}\right)<-x\right) \gamma_{1}(\mathrm{~d} x)\right\} \\
& +\zeta_{1}^{4}\left\{\int_{\phi_{0}}^{\infty} P\left(\tilde{\varphi}_{1}\left(G_{3}\right)>-x\right) \gamma_{1}(\mathrm{~d} x)+\int_{-\infty}^{\phi_{0}} P\left(\tilde{\varphi}_{1}\left(G_{4}\right)<-x\right) \gamma_{1}(\mathrm{~d} x)\right\}+\cdots+\zeta_{1}^{j}\left\{\int_{\phi_{0}}^{\infty} P\left(\tilde{\varphi}_{1}\left(G_{j}\right)>-x\right) \gamma_{1}(\mathrm{~d} x)\right. \\
& \left.+\int_{-\infty}^{\phi_{0}} P\left(\tilde{\varphi}_{1}\left(G_{j-1}\right)<-x\right) \gamma_{1}(\mathrm{~d} x)\right\}+\zeta_{1}^{j+1}\left\{\int_{\phi_{0}}^{\infty} P\left(\tilde{\varphi}_{1}\left(G_{j+1}\right)>-x\right) \gamma_{1}(\mathrm{~d} x)+\int_{-\infty}^{\phi_{0}} P\left(\tilde{\varphi}_{1}\left(G_{j}\right)<-x\right) \gamma_{1}(\mathrm{~d} x)\right\} \\
& +\zeta_{1}^{j+2}\left\{\int_{\phi_{0}}^{\infty} P\left(\varphi_{1}\left(G_{j+2}\right)>-x\right) \gamma_{1}(\mathrm{~d} x)+\int_{-\infty}^{\phi_{0}} P\left(\tilde{\varphi}_{1}\left(G_{j+1}\right)<-x\right) \gamma_{1}(\mathrm{~d} x)\right\} \\
& \left.+\zeta_{1}^{j+3}\left\{\int_{\phi_{0}}^{\infty} P\left(\varphi_{1}\left(G_{j+3}\right)>-x\right) \gamma_{1}(\mathrm{~d} x)+\int_{-\infty}^{\phi_{0}} P\left(\tilde{\varphi}_{1}\left(G_{j+2}\right)<-x\right) \gamma_{1}(\mathrm{~d} x)\right\}+\cdots\right] \\
& +\lambda_{2}\left(\zeta_{2}-1\right)\left[P\left(\tau_{\bar{\phi}}>0\right)+\zeta_{2}\left(\tau_{\bar{\phi}}>\bar{G}_{1}\right)+\zeta_{2}^{2}\left\{\int_{\bar{\phi}_{0}}^{\infty} P\left(\tilde{\varphi}_{2}\left(\bar{G}_{1}\right)>-y\right) \gamma_{2}(\mathrm{~d} y)\right.\right. \\
& \left.+\int_{-\infty}^{\bar{\phi}_{0}} P\left(\tilde{\varphi}_{2}\left(\bar{G}_{2}\right)<-y\right) \gamma_{2}(\mathrm{~d} y)\right\}+\zeta_{2}^{3}\left\{\int_{\bar{\phi}_{0}}^{\infty} P\left(\tilde{\varphi}_{2}\left(\bar{G}_{3}\right)>-y\right) \gamma_{2}(\mathrm{~d} y)+\int_{-\infty}^{\bar{\phi}_{0}} P\left(\tilde{\varphi}_{2}\left(\bar{G}_{2}\right)<-y\right) \gamma_{2}(\mathrm{~d} y)\right\}+\cdots \\
& +\zeta_{2}^{j}\left\{\int_{\bar{\phi}_{0}}^{\infty} P\left(\tilde{\varphi}_{2}\left(\bar{G}_{j}\right)>-y\right) \gamma_{2}(\mathrm{~d} y)+\int_{-\infty}^{\bar{\phi}_{0}} P\left(\tilde{\varphi}_{2}\left(\bar{G}_{j-1}\right)<-y\right) \gamma_{2}(\mathrm{~d} y)\right\}+\zeta_{2}^{j+1}\left\{\int_{\bar{\phi}_{0}}^{\infty} P\left(\tilde{\varphi}_{2}\left(\bar{G}_{j+1}\right)>-y\right) \gamma_{2}(\mathrm{~d} y)\right. \\
& \left.+\int_{-\infty}^{\phi_{0}} P\left(\tilde{\varphi}_{2}\left(\bar{G}_{j}\right)<-y\right) \gamma_{2}(\mathrm{~d} y)\right\}+\zeta_{2}^{j+2}\left\{\int_{\bar{\phi}_{0}}^{\infty} P\left(\varphi_{2}\left(\bar{G}_{j+2}\right)>-y\right) \gamma_{2}(\mathrm{~d} y)+\int_{-\infty}^{\bar{\phi}_{0}} P\left(\tilde{\varphi}_{2}\left(\bar{G}_{j+1}\right)<-y\right) \gamma_{2}(\mathrm{~d} y)\right\} \\
& \left.+\zeta_{2}^{j+3}\left\{\int_{\bar{\phi}_{0}}^{\infty} P\left(\varphi_{2}\left(\bar{G}_{j+3}\right)>-y\right) \gamma_{2}(\mathrm{~d} y)+\int_{-\infty}^{\bar{\phi}_{0}} P\left(\tilde{\varphi}_{2}\left(\bar{G}_{j+2}\right)<-y\right) \gamma_{2}(\mathrm{~d} y)\right\}+\cdots\right]
\end{aligned}
$$

Then 


$$
\begin{aligned}
E\left(\tau_{\hat{\phi}}\right) \leq & \lambda_{1}\left(\zeta_{1}-1\right)\left[g_{1}+\left(\zeta_{1}+1\right) \zeta_{1}^{2} \int_{-\infty}^{\phi_{0}} P\left(\tilde{\varphi}_{1}\left(G_{2}\right)<-x\right) \gamma_{1}(\mathrm{~d} x)\right. \\
& +\left(\zeta_{1}+1\right) \zeta_{1}^{4} \int_{-\infty}^{\phi_{0}} P\left(\tilde{\varphi}_{1}\left(G_{4}\right)<-x\right) \gamma_{1}(\mathrm{~d} x)+\cdots+\left(\zeta_{1}+1\right) \zeta_{1}^{j+1} \int_{-\infty}^{\phi_{0}} P\left(\tilde{\varphi}_{1}\left(G_{j+1}\right)<-x\right) \gamma_{1}(\mathrm{~d} x)+\cdots \\
& +\left(\zeta_{1}+1\right) \zeta_{1}^{3} \int_{\phi_{0}}^{\infty} P\left(\tilde{\varphi}_{1}\left(G_{3}\right)>-x\right) \gamma_{1}(\mathrm{~d} x)+\left(\zeta_{1}+1\right) \zeta_{1}^{5} \int_{\phi_{0}}^{\infty} P\left(\tilde{\varphi}_{1}\left(G_{5}\right)>-x\right) \gamma_{1}(\mathrm{~d} x)+\cdots \\
& \left.+\left(\zeta_{1}+1\right) \zeta_{1}^{j} \int_{\phi_{0}}^{\infty} P\left(\tilde{\varphi}_{1}\left(G_{j}\right)>-x\right) \gamma_{1}(\mathrm{~d} x)+\cdots+\left(\zeta_{1}+1\right) \zeta_{1}^{j+2} \int_{\phi_{0}}^{\infty} P\left(\tilde{\varphi}_{1}\left(G_{j+2}\right)>-x\right) \gamma_{1}(\mathrm{~d} x)+\cdots\right] \\
& +\lambda_{2}\left(\zeta_{2}-1\right)\left[\bar{g}_{2}+\left(\zeta_{2}+1\right) \zeta_{2}^{2} \int_{-\infty}^{\phi_{0}} P\left(\tilde{\varphi}_{2}\left(\bar{G}_{2}\right)<-y\right) \gamma_{2}(\mathrm{~d} y)+\left(\zeta_{2}+1\right) \zeta_{2}^{4} \int_{-\infty}^{\bar{\phi}_{0}} P\left(\tilde{\varphi}_{2}\left(\bar{G}_{4}\right)<-y\right) \gamma_{2}(\mathrm{~d} y)+\cdots\right. \\
& +\left(\zeta_{2}+1\right) \zeta_{2}^{j+2} \int_{-\infty}^{\bar{\phi}_{0}} P\left(\tilde{\varphi}_{2}\left(\bar{G}_{j+2}\right)<-y\right) \gamma_{2}(\mathrm{~d} y)+\cdots+\left(\zeta_{2}+1\right) \zeta_{2}^{3} \int_{\bar{\phi}_{0}}^{\infty} P\left(\tilde{\varphi}_{2}\left(\bar{G}_{3}\right)>-y\right) \gamma_{2}(\mathrm{~d} y) \\
& +\left(\zeta_{2}+1\right) \zeta_{2}^{5} \int_{\bar{\phi}_{0}}^{\infty} P\left(\tilde{\varphi}_{2}\left(\bar{G}_{5}\right)>-y\right) \gamma_{2}(\mathrm{~d} y)+\cdots+\left(\zeta_{2}+1\right) \zeta_{2}^{j} \int_{\bar{\phi}_{0}}^{\infty} P\left(\tilde{\varphi}_{2}\left(\bar{G}_{j}\right)>-y\right) \gamma_{2}(\mathrm{~d} y)+\cdots \\
& \left.+\left(\zeta_{2}+1\right) \zeta_{2}^{j+2} \int_{\bar{\phi}_{0}}^{\infty} P\left(\tilde{\varphi}_{2}\left(\bar{G}_{j+2}\right)>-y\right) \gamma_{2}(\mathrm{~d} y)+\cdots\right] \\
& +\lambda_{2}\left(\zeta_{2}-1\right)\left[\bar{g}_{1}\left(\zeta_{2}+1\right)\left\{\int_{-\infty} \int_{\bar{\phi}_{0}} \bar{m}_{1}(y) \gamma_{2}(\mathrm{~d} y)+\int_{\bar{\phi}_{0}}^{\infty} \bar{q}_{1}(y) \gamma_{2}(\mathrm{~d} y)+\int_{\bar{\phi}_{0}}^{\infty} \overline{\tilde{q}}_{1}(y) \gamma_{2}(\mathrm{~d} y)\right\}\right] \\
& E\left(\lambda_{1}\left(\zeta_{1}-1\right)\left[g_{1}\left(\zeta_{1}+1\right)\left\{\int_{-\infty}^{\phi_{0}} m_{1}(x) \gamma_{1}(\mathrm{~d} x)+\int_{\phi_{1}}^{\infty}(x) \gamma_{1}(\mathrm{~d} x)+\int_{\phi_{1}}^{\infty} \tilde{q}_{1}(x) \gamma_{1}(\mathrm{~d} x)\right\}\right]\right.
\end{aligned}
$$

where

$$
\begin{gathered}
g_{1}=P\left(\tau_{\phi}>0\right)+\zeta_{1} P\left(\tau_{\phi}>G_{1}\right)+\zeta_{1}^{2} \int_{\phi_{0}}^{\infty} P\left(\tilde{\varphi}_{1}\left(G_{1}\right)>-x\right) \gamma_{1}(\mathrm{~d} x) \\
\bar{g}_{1}=P\left(\tau_{\bar{\phi}}>0\right)+\zeta_{2} P\left(\tau_{\bar{\phi}}>\bar{G}_{1}\right)+\zeta_{2}^{2} \int_{\bar{\phi}_{0}}^{\infty} P\left(\tilde{\varphi}_{2}\left(\bar{G}_{1}\right)>-y\right) \gamma_{2}(\mathrm{~d} y) \\
m_{1}(x)=\zeta_{1}^{2} P\left(\tilde{\varphi}_{1}\left(G_{2 i}\right)<-x\right), \\
\bar{m}_{1}(y)=\zeta_{2}^{2} P\left(\tilde{\varphi}_{2}\left(\bar{G}_{2 i}\right)<-y\right), \\
q_{1}(x)=\sum_{j=1}^{(j-1) / 2} \zeta_{1}^{2 i+1} P\left[\tilde{\varphi}_{1}\left(G_{2 j+1}\right)>-x\right] \\
\bar{q}_{1}(y)=\sum_{i=1}^{(j-1) / 2} \zeta_{1}^{2 i+1} P\left[\tilde{\varphi}_{2}\left(\bar{G}_{2 i+1}\right)>-y\right] \\
\tilde{q}_{1}(x)=\sum_{i=(j+1) / 2}^{\infty} \zeta_{2}^{2 i+1} P\left[\varphi_{1}\left(G_{2 i+1}\right)>-x\right]
\end{gathered}
$$

and

$$
\overline{\tilde{q}}_{1}(y)=\sum_{i=(j+1) / 2}^{\infty} \zeta_{2}^{2 j+1} P\left[\varphi_{2}\left(\bar{G}_{2 i+1}\right)>-y\right]
$$

the other cases can be proved by similar way 
Lemma 2.2. Let $a_{n} \geq 0$ for $n \geq 0$, and $a_{n+1} \leq a_{n},\left\{d_{n}\right\} n \geq 0$ be a strictly increasing sequence of integers with $d_{0}$ $=0$ then for any $k \leq 0$,

$$
\sum_{n=k}^{\infty}\left[d_{n+1}-d_{n}\right] a_{d_{n+1}} \leq \sum_{n=d_{k}}^{\infty} a_{n} \leq \sum_{n=k}^{\infty}\left[d_{n+1}-d_{n}\right] a_{d_{n}} \quad \text { (see EL-Rayes et al. [5]) }
$$

Theorem 2.2. The chosen search plan satisfies

$$
\begin{gathered}
\tilde{q}_{2}(x) \leq L(|x|), \overline{\tilde{q}}_{2}(y) \leq L(|x|) \\
q_{2}(x) \leq L^{\prime}(|x|), \bar{q}_{2}(y) \leq L^{\prime}(|y|), \phi_{0}, \bar{\phi}_{0}>0 \\
m_{1}(x) \leq L^{\prime \prime}(|x|), \bar{m}_{1}(y) \leq L^{\prime \prime}(|y|) \\
\tilde{q}_{1}(x) \leq L^{\prime \prime \prime}(|x|), \overline{\tilde{q}}_{1}(x) \leq L^{\prime \prime \prime}(|y|), \phi_{0}, \bar{\phi}_{0}<0
\end{gathered}
$$

where $L(|x|), L^{\prime}(|x|), L^{\prime \prime}(|x|), L^{\prime \prime \prime}(|x|), L(y), L^{\prime}(|y|), L^{\prime \prime}(|y|), L^{\prime \prime \prime}(|y|)$ are linear functions.

Proof. We shall prove the theorem for $\tilde{q}_{2}(x)$ and $\overline{\tilde{q}}_{2}(y)$, since the other cases can be proved by a similar way.

$$
\begin{aligned}
& \tilde{q}_{2}(x)=\sum_{i=1}^{\infty} \zeta_{1}^{2 i+1} P\left[\varphi\left(G_{2 i+1}\right)>-x\right], \quad \phi_{0}>0, \\
& \overline{\tilde{q}}_{2}(y)=\sum_{i=1}^{\infty} \zeta_{2}^{2 i+1} P\left[\varphi\left(\bar{G}_{2 i+1}\right)>-y\right], \quad \bar{\phi}_{0}>0
\end{aligned}
$$

(i) if $x \geq \phi_{0}$

$$
\tilde{q}_{2}(x)=\tilde{q}_{2}\left(\phi_{0}\right)+\sum_{i=1}^{\infty} \zeta_{1}^{2 i+1} P\left[-x<\varphi\left(G_{2 i+1}\right) \leq-\phi_{0}\right]
$$

and $y \geq \bar{\phi}_{0}$

$$
\overline{\tilde{q}}_{2}(y)=\overline{\tilde{q}}_{2}\left(\bar{\phi}_{0}\right)+\sum_{i=1}^{\infty} \zeta_{2}^{2 i+1} P\left[-y<\varphi\left(\bar{G}_{2 i+1}\right) \leq-\bar{\phi}_{0}\right]
$$

(ii) if $0 \leq x \leq \phi_{0}$

$$
\tilde{q}_{2}(x)=\tilde{q}_{2}(0)+\sum_{i=1}^{\infty} \zeta_{1}^{2 i+1} P\left[-x<\varphi\left(G_{2 i+1}\right) \leq 0\right]
$$

and $0 \leq y \leq \bar{\phi}_{0}$

$$
\overline{\tilde{q}}_{2}(y)=\overline{\tilde{q}}_{2}(0)+\sum_{i=1}^{\infty} \zeta_{2}^{2 i+1} P\left[-y<\varphi\left(\bar{G}_{2 i+1}\right) \leq 0\right]
$$

(iii) if $x \leq 0$

$$
\tilde{q}_{2}(x)=\tilde{q}_{2}(0)-\sum_{i=1}^{\infty} \zeta_{1}^{2 i+1} P\left[0<\varphi\left(G_{2 i+1}\right) \leq-x\right]
$$

and $y \leq 0$

$$
\overline{\tilde{q}}_{2}(y)=\overline{\tilde{q}}_{2}(0)-\sum_{i=1}^{\infty} \xi_{2}^{2 i+1} P\left[0<\varphi\left(\bar{G}_{2 i+1}\right) \leq-y\right]
$$

we have $x \geq \phi_{0}$ and $y \geq \bar{\phi}_{0}$ in (ii)

$$
\begin{gathered}
\tilde{q}_{2}(x) \leq \tilde{q}_{2}(0) \text { and } \overline{\tilde{q}}_{2}(y) \leq \overline{\tilde{q}}_{2}(0) \\
\tilde{q}_{2}(x)=\tilde{q}_{2}\left(\phi_{0}\right)+\sum_{i=1}^{\infty} \zeta_{1}^{2 i+1} P\left[-x<\varphi\left(G_{2 i+1}\right) \leq-\phi_{0}\right]
\end{gathered}
$$




$$
\overline{\tilde{q}}_{2}(y)=\overline{\tilde{q}}_{2}\left(\bar{\phi}_{0}\right)+\sum_{i=1}^{\infty} \zeta_{2}^{2 i+1} P\left[-y<\varphi\left(\bar{G}_{2 i+1}\right) \leq-\bar{\phi}_{0}\right]
$$

but,

$$
\begin{aligned}
& \tilde{q}_{2}(x)=\tilde{q}_{2}(0)+\sum_{i=1}^{\infty} \zeta_{1}^{2 i+1} P\left[-x<\varphi\left(G_{2 i+1}\right)<0\right] \\
& \overline{\tilde{q}}_{2}(y)=\overline{\tilde{q}}_{2}(0)+\sum_{i=1}^{\infty} \zeta_{2}^{2 i+1} P\left[-y<\varphi\left(\bar{G}_{2 i+1}\right)<0\right]
\end{aligned}
$$

from [1]

$$
\begin{gathered}
\tilde{q}_{2}(x)=\sum_{i=1}^{\infty} \zeta_{1}^{2 i+1} P\left[\varphi\left(G_{2 i+1}\right)>0\right] \leq \sum_{i=1}^{\infty} \zeta_{1}^{2 i+1} \varepsilon^{G_{2 i+1}} \leq \frac{\zeta_{1}^{3}}{\zeta_{1}^{2}-1} \\
\overline{\tilde{q}}_{2}(y)=\sum_{i=1}^{\infty} \zeta_{2}^{2 i+1} P\left[\varphi\left(\bar{G}_{2 i+1}\right)>0\right] \leq \sum_{i=1}^{\infty} \zeta_{2}^{2 i+1} \varepsilon^{\bar{G}_{2 i+1}} \leq \frac{\zeta_{2}^{3}}{\zeta_{2}^{2}-1}, \quad 0<\varepsilon<1
\end{gathered}
$$

we define the following

$$
\text { 1) } \begin{aligned}
d_{n} & =G_{2 n+1}=\left(\zeta_{1}^{2 n+1}-1\right) \\
\bar{d}_{n} & =\bar{G}_{2 n+1}=\left(\zeta_{2}^{2 n+1}-1\right)
\end{aligned}
$$

2) $k(n)=\varphi(n)=\sum_{i=1}^{n} W_{i}$, where $\left\{W_{i}\right\}_{i \geq 0}$ is sequence of (i. i. d. r. v.) $\bar{k}(n)=\bar{\varphi}(n)=\sum_{i=1}^{n} \bar{W}_{i}$ where $\left\{\bar{W}_{i}\right\}_{i \geq 0}$ is sequence of (i. i. d. r. v.)

3) $a(n)=P[-x<k(n) \leq 0]=\sum_{j=0}^{[|x|]]} P[-(j+1)<k(n) \leq-j]$

$$
\bar{a}(n)=P[-y<\bar{k}(n) \leq 0]=\sum_{j=0}^{[y \mid]} P[-(j+1)<\bar{k}(n) \leq-j]
$$

4) $n_{1}$ is an integer such that $d_{n_{1}}=b_{1}|x|+b_{2} ; n_{2}$ is an integer such that $d_{n_{2}}=\bar{b}_{1}|x|+\bar{b}_{2}$

5) $U(j, j+1)=\sum_{n=0}^{\infty} t_{2} P[-(j+1)<k(n) \leq-j]$

$$
\bar{U}(j, j+1)=\sum_{n=0}^{\infty} t_{2} P[-(j+1)<\bar{k}(n) \leq-j]
$$

6) $\alpha_{1}=\zeta_{1}^{2} /\left(\zeta_{1}^{2}-1\right)$

$$
\alpha_{2}=\zeta_{2}^{2} /\left(\zeta_{2}^{2}-1\right)
$$

If $n>d_{n}$ and $\bar{d}_{n}$ then $a_{(n)}, \bar{a}_{(n)}$ are non-increasing, see [15], and we can apply Lemma 2.2 in suitable steps.

$$
\begin{aligned}
\tilde{q}_{2}(x)-\tilde{q}_{2}(0)=\sum_{i=1}^{\infty} \zeta_{1}^{2 i+1} P\left[-x<\varphi\left(G_{2 i+1}\right) \leq-0\right] & =\sum_{n=1}^{n_{1}} \zeta_{1}^{2 n+1} a\left(d_{n}\right)+\sum_{n=n_{1}+1}^{\infty} \zeta_{1}^{2 n+1} a\left(d_{n}\right) \\
& \leq \sum_{n=1}^{n_{1}} \zeta_{1}^{2 n+1}+\alpha_{1} \sum_{n=n_{1}+1}^{\infty}\left(d_{n}-d_{n-1}\right) a\left(d_{n}\right) \\
& \leq \sum_{n=1}^{n_{1}} \zeta_{1}^{2 n+1}+\alpha_{1} \sum_{n=d_{n_{1}}}^{\infty} a(n) \\
& \leq \sum_{n=1}^{n_{1}} \zeta_{1}^{2 n+1}+\alpha_{1} \sum_{j=0}^{|x| / 2} U(j, j+1)
\end{aligned}
$$

and 


$$
\begin{aligned}
\overline{\tilde{q}}_{2}(y)-\overline{\tilde{q}}_{2}(0)=\sum_{i=1}^{\infty} \zeta_{2}^{2 i+1} P\left[-y<\varphi\left(\bar{G}_{2 i+1}\right) \leq 0\right] & =\sum_{n=1}^{n_{2}} \zeta_{2}^{2 n+1} a\left(\bar{d}_{n}\right)+\sum_{n=n_{2}+1}^{\infty} \zeta_{2}^{2 n+1} \bar{a}\left(\bar{d}_{n}\right) \\
& \leq \sum_{n=1}^{n_{2}} \zeta_{2}^{2 n+1}+\alpha_{2} \sum_{n=n_{2}+1}^{\infty} \zeta_{2}^{2 n+1} \bar{a}\left(\bar{d}_{n}\right) \\
& \leq \sum_{n=1}^{n_{2}} \zeta_{2}^{2 n+1}+\alpha_{2} \sum_{n=n_{2}+1}^{\infty}\left(\bar{d}_{n}-\bar{d}_{n-1}\right) \bar{a}(n) \\
& \leq \sum_{n=1}^{n_{2}} \zeta_{2}^{2 n+1}+\alpha_{2} \sum_{n=d_{n}}^{\infty} \bar{a}(n) \\
& \leq \sum_{n=1}^{n_{2}} \zeta_{2}^{2 n+1}+\alpha_{2} \sum_{j=0}^{\mid y / 2} \bar{U}(j, j+1)
\end{aligned}
$$

Since $U(j, j+1), \bar{U}(j, j+1)$ satisfies the conditions of renwal theorem (see [15]) hence $U(j, j+1)$, $\bar{U}(j, j+1)$ is bounded for all $\mathrm{j}$ by a constant, so

$$
\begin{gathered}
\tilde{q}_{2}(x) \leq \tilde{q}_{2}\left(\phi_{0}\right)+M_{1}+M_{2}|x|=L(|x|) \\
\overline{\tilde{q}}_{2}(y) \leq \overline{\tilde{q}}_{2} L\left(\bar{\phi}_{0}\right)+\bar{M}_{1}+\bar{M}_{2}|y|=L(|y|)
\end{gathered}
$$

Theorem 2.3. If there exist a finite search plan $(\phi, \bar{\phi}) \in \hat{\Phi}$ then $E\left|Z_{0}\right|$ is finite.

Proof. If $E \tau_{\hat{\phi}}<\infty$, then $P\left(\tau_{\phi}<\infty\right)=1$ or $p\left(\tau_{\bar{\phi}}<\infty\right)=1$

then $P\left(\tau_{\phi}<\infty\right)+\left(\tau_{\bar{\phi}}<\infty\right)=1$, that is $Z_{0}=\phi\left(\tau_{\hat{\phi}}\right)-S\left(\tau_{\hat{\phi}}\right)$ with probability 1 , so

$$
\left|Z_{o}\right|=\phi\left(\tau_{\hat{\phi}}\right)+\left|S\left(\tau_{\hat{\phi}}\right)\right| \leq \tau_{\hat{\phi}}+\left|S\left(\tau_{\hat{\phi}}\right)\right|
$$

Hence $E\left|Z_{0}\right| \leq E \tau_{\hat{\phi}}+E\left|S\left(\tau_{\hat{\phi}}\right)\right|$, but $\left|S\left(\tau_{\hat{\phi}}\right)\right| \leq \tau_{\hat{\phi}}$ then $E\left|S\left(\tau_{\hat{\phi}}\right)\right| \leq E \tau_{\hat{\phi}}$ and $E\left|Z_{0}\right|<\infty$.

Remark A direct consequence of theorems 1,2 and 3 in Section 2 is the existence of a finite search plan $\hat{\phi} \in \hat{\Phi}(t)$ if and only if $E\left|Z_{0}\right| \leq \infty$, and then the set of search plans, which defined in Section 2, satisfies the conditions of Theorem 1 if the expectation value of initial position of the lost target is finite.

\section{Existence of an Optimal Path}

Definition. Let $\hat{\phi}_{n} \in \hat{\Phi}(t)$ be a sequence of search plans, we say that $\hat{\phi}_{n}$ converges to $\hat{\phi}$ as $n$ tends to $\infty$ if for any $t \in I^{+} \rightarrow, \hat{\phi}_{n}(t)$ converges to $\hat{\phi}(t)$ on every compact subset.

Theorem 3.1. Let for any $t \in \stackrel{+}{\mathfrak{R}}, S(t)$ be a process with continuous sample paths. The mapping $(\phi, \bar{\phi}) \longrightarrow E\left(\tau_{\widehat{\Phi}}\right) \in \mathfrak{R}^{+}$is lower semi-continuous on $\widehat{\Phi}(t)$.

Proof. Let $\xi$ be a sample point on $L_{1}$ corresponding to the sample path $\xi(t)$ of $X_{o}+S(t)$ and $\dot{\xi}$ be a sample point on $L_{2}$ corresponding to the sample path $\dot{\xi}(t)$ of $Y_{o}+S(t)$. Let $\left\{\phi_{n}\right\}_{n \geq 1}$ be a sequence of search paths which converges to $\phi \in \Phi_{V_{1}}(t)$ and $\left\{\bar{\phi}_{n}\right\}_{n \geq 1}$ converges to $\bar{\phi} \in \Phi_{V_{2}}(t)$. Given $t \in \mathfrak{R}^{+}$, we define for any $n \geq 1$

$$
\begin{aligned}
& B_{n}(t)=\left\{\xi: \min _{0 \leq x \leq t}\left|\xi(x)-\phi_{n}(x)\right|>0\right\}, \\
& B(t)=\left\{\xi: \min _{0 \leq x \leq t}|\xi(x)-\phi(x)|>0\right\}, \\
& \bar{B}_{n}(t)=\left\{\bar{\xi}: \min _{0 \leq y \leq t}\left|\bar{\xi}(y)-\bar{\phi}_{n}(y)\right|>0\right\} \\
& \text { and } \bar{B}(t)=\left\{\bar{\xi}: \min _{0 \leq y \leq t}|\bar{\xi}(y)-\bar{\phi}(y)|>0\right\}
\end{aligned}
$$


Let $\xi \in B(t), \bar{\xi} \in \bar{B}(t)$ and since $\left\{\phi_{n}\right\}_{n \geq 1}$ converges uniformly on $[0, t]$ to $\phi$, then there exists an integer $n(\xi)$ such that for all $n \geq n(\xi)$ and for any $0 \leq x \leq t$

$$
\left|\phi_{n}(x)-\phi(x)\right|<\varepsilon=0.5 \min _{0 \leq x \leq t}|\xi(x)-\phi(x)|
$$

and $|\xi(x)-\phi(x)| \leq\left|\xi(x)-\phi_{n}(x)\right|+\left|\phi(x)-\phi_{n}(x)\right|$, then $\left|\xi(x)-\phi_{n}(x)\right| \geq|\xi(x)-\phi(x)|-\left|\phi(x)-\phi_{n}(x)\right| \geq 2 \varepsilon-\varepsilon=\varepsilon>0$.

Hence $\xi \in B_{n}(t)$ for all $n>n(\xi)$ and so $B(t) \subset \lim _{n \rightarrow \infty} \inf B_{n}(t)$,

$$
\int_{0}^{\infty} P(B(t)) \mathrm{d} t \leq \int_{0}^{\infty} P\left(\lim _{n \rightarrow \infty} \inf B_{n}(t)\right) \mathrm{d} t \leq \int_{0}^{\infty} \lim _{n \rightarrow \infty} \inf P\left(B_{n}(t)\right) \mathrm{d} t
$$

by the same way we can get

$$
\int_{0}^{\infty} P(\bar{B}(t)) \mathrm{d} t \leq \int_{0}^{\infty} P\left[\lim _{n \rightarrow \infty} \inf \bar{B}_{n}(t)\right] \mathrm{d} t \leq \int_{0}^{\infty} \lim _{n \rightarrow \infty} \inf P\left(\bar{B}_{n}(t)\right) \mathrm{d} t
$$

since the sample paths are continuous then

$$
B_{n}(t)=\left\{\tau_{\phi_{n}} \succ t\right), \quad B(t)=\left\{\tau_{\phi} \succ t\right\}, \quad \bar{B}_{n}(t)=\left\{\tau_{\bar{\phi}_{n}} \succ t\right\} \text { and } B=\left\{\tau_{\bar{\phi}} \succ t\right\}
$$

we get $\int_{0}^{\infty} p\left(\tau_{\phi}>t\right) \mathrm{d} t \leq \int_{\infty}^{\infty} \lim _{n \rightarrow \infty} \inf p\left(\tau_{\phi_{n}}>t\right) \mathrm{d} t$ and $\int_{0_{\infty}}^{\infty} p\left(\tau_{\bar{\phi}}>t\right) \mathrm{d} t \leq \int_{0}^{\infty} \lim _{n \rightarrow \infty} \inf p\left(\tau_{\bar{\phi}_{n}}>t\right) \mathrm{d} t$, we obtain

$$
\int_{0}^{\infty}\left[p\left(\tau_{\bar{\phi}}>t\right)+p\left(\tau_{\phi}>t\right)\right] \mathrm{d} t \leq \int_{0}^{0_{n}^{\infty}} \liminf _{n \rightarrow \infty}\left[p\left(\tau_{\bar{\phi}_{n}}>t\right)+p\left(\tau_{\bar{\phi}_{n}}>t\right)\right] \mathrm{d} t
$$

then

$$
\int_{0}^{\infty} p(\tau>t) \mathrm{d} t \leq \int_{0}^{\infty} \lim _{n \rightarrow \infty} \inf p\left(\tau_{\Phi_{n}}>t\right) \mathrm{d} t
$$

where $\widehat{\Phi}_{n}=\left(\phi_{n}, \bar{\phi}_{n}\right)$

hence $E\left(\tau_{\hat{\Phi}}\right) \leq E\left(\lim _{n \rightarrow \infty} \inf \tau_{\widehat{\Phi}_{n}}\right)$. By Fatou lemma, we get

$$
E\left(\tau_{\widehat{\Phi}}\right) \leq E\left(\lim _{n \rightarrow \infty} \inf \tau_{\widehat{\Phi}_{n}}\right) \leq \lim _{n \rightarrow \infty} \inf E\left(\tau_{\widehat{\Phi}_{n}}\right) .
$$

Since $\Phi$ is sequentially compact (see [2]), then by the same way $\bar{\Phi}$ is sequentially compact. It is known that a lower semi-continuous function over a sequentially compact space attains its minimum.

\section{Conclusion}

We consider, here, the search for a lost target on one of two disjoint lines, where the target moves randomly according to continuous stochastic process which satisfies some conditions. Theorems conclude that there exists a finite search plan if and only if the expectation value of the initial position of the target is finite. Existence of optimal search plan is proved.

\section{Acknowledgements}

The author would like to thank the reviewers and Editorial Board of AM Journal for their helpful suggestions which would improve the article.

\section{References}

[1] Mohamed, Abd El-M.A. (2005) Generalized Search for One Dimensional Random Walker. International Journal of Pure and Applied Mathematics, 19, 375-387.

[2] Ohsumi, A. (1991) Optimal Search for a Markovian Target. Naval Research Logistics, 38, 531-554. http://dx.doi.org/10.1002/1520-6750(199108)38:4<531::AID-NAV3220380407>3.0.CO;2-L

[3] El-Rayes, A.B. and Abd EL-Moneim, M.A. (1989) Searching for a Randomly Moving Target. Proceeding of the Third ORMA Conference (Germany), 1, 323-329. 
[4] Washburn, A.R. (1989) Search and Detection. 2nd Edition, ORSA Books, Arlington.

[5] El-Rayes, A.B., Abd-Elmoneim, M.A. and Abu Gabl, H.M. (2003) A Linear Search for a Brownian Target Motion. Acta Mathematica Scienta, 23B, 321-327.

[6] Balkhi, Z.T. (1989) Generalized Optimal Search Paths for Continuous Univariate Random Variables. Recherche Operationnelle Operation Research, 23, 67-96.

[7] El-Rayes, A.B. and Abd-Elmoneim, M.A. (1998) Oscillating Search for a Randomly Located. AMSE, 40, $29-40$.

[8] El-Rayes, A.B., Abd-ELmoneim, M.A. and Fergani, H. (1993) On the Generalized Linear Search Problem. Delta Journal, 6, 1-10.

[9] Balkhi, Z.T. (1987) The Generalized Linear Search Problem, Existence of Optimal Search Paths. Journal of the Operations Research Society of Japan, 30, 399-420.

[10] Rousseeuw, P. (1983) Optimal Search Paths for Random Variables. Journal of Computational and Applied Mathematics, 9, 279-286. http://dx.doi.org/10.1016/0377-0427(83)90020-1

[11] Mohamed, Abd El-M.A. and AbuGabl, H.M. (2000) Generalized Optimal Search Paths for a Randomly Located Target. Annual Conference (Cairo) ISSR, Math. Statistics Part, 35, 17-29.

[12] Stone, L.D. (1975) Theory of Optimal Search. Academic Press, New York.

[13] Fristedt, B. and Heath, D. (1974) Searching for a Particle on the Real Line. Advances in Applied Probability, 6, 79-102. http://dx.doi.org/10.2307/1426208

[14] Mohamed, Abd El-M.A., Kassem, M.A. and El-Hadidy, M.A. (2011) Multiplicative Linear Search for Brownian Target Motion. Applied Mathematical Modelling, 35, 4127-4139. http://dx.doi.org/10.1016/j.apm.2011.03.024

[15] Mohamed, Abd El-M.A. and AbuGabl, H.M. (2008) Double Linear Search Problem for a Brownian Motion. Journal of the Egyptian Mathematical Society, 16, 99-107. 